\title{
Interprofessional Education digital implementation
}

\author{
Victorio Burcio-Martin \\ AUT University \\ victorio.burciomartin@aut.ac.nz
}

Submission Type: Case Study (20mins + 10mins Q\&A)

Keywords: Interprofessional teaching, Digital implementation.

In 2017, AUT rolled out a new initiative called the Interprofessional Learning Zone (ILZ). ILZ is a central, student-driven programme that provides all clinical students (regardless of the programme they are enrolled in) with an opportunity to study and learn together (Flood, 2017) - student enrolments will total to over 1000 in a year. ILZ is a self-select programme, where students volunteer and complete ILZ units to get extra credits and recognition. ILZ as such is not a formal qualification, rather a 'clinical passport' that provides the students with industry relevant knowledge and understanding with a key focus on enabling a shared understanding of clinical practices (Floyd \& Morrison, 2014).

ILZ being student-driven means the learning path and process in the programme is determined by the students. The students enrol and complete learning tasks and activities according to the time they have and their learning needs.

The student-driven nature of the programme created significant challenges when it came to evaluating the use of technologies that could help facilitate the course. It was uncertain how many students would enrol and complete a unit of study in ILZ. This created significant pedagogical issues, such as if a group of students enrolled to undertake an ILZ unit how would bookings for the simulation labs be handled? In particular, the system needed to ensure a mix of students from all disciplines to create opportunities for interprofessional learning.

This presentation will provide an overview of the evaluative and design processes that informed the choice of the technology in ILZ - discussing similar issues as above encountered over a period of time.

\section{References}

Flood, B. (2017). Toward a spirit of interprofessional practice: A hermeneutic phenomenological study Thesis type: Doctoral Thesis. Auckland University of Technology. http://hdl.handle.net/10292/10776

Floyd, A., \& Morrison, M. (2014). Exploring identities and cultures in inter-professional education and collaborative professional practice. Studies in Continuing Education, 36(1), 38-53. http://doi.org/10.1080/0158037X.2013.783472 